\title{
Quaderni
}

QUADERNI Communication, technologies, pouvoir

79 | Automne 2012

Produire la démocratie

\section{Publics « forts » et «faibles » du web : le cas des consultations permanentes de la Commission Européenne}

Romain Badouard

\section{(2) OpenEdition \\ Journals}

Édition électronique

URL : http://journals.openedition.org/quaderni/626

DOI : 10.4000/quaderni.626

ISSN : 2105-2956

Éditeur

Les éditions de la Maison des sciences de l'Homme

\section{Édition imprimée}

Date de publication : 5 octobre 2012

Pagination : 99-108

Référence électronique

Romain Badouard, «Publics «forts » et «faibles » du web : le cas des consultations permanentes de la Commission Européenne », Quaderni [En ligne], 79 | Automne 2012, mis en ligne le 05 octobre 2014 consulté le 02 mai 2019. URL : http://journals.openedition.org/quaderni/626 ; DOI : 10.4000/ quaderni.626 


\section{Politique}

\section{Publics "forts" et "faibles"}

du web : le cas des consultations permanentes de la Commission Européenne

\section{Romain Badouard}

COSTECH/UTC
Les pratiques politiques en ligne sont l'objet d'une littérature académique abondante. Parmi la grande diversité des thématiques abordées dans ces travaux, l'analyse du rapport que les publics qui se forment sur le web entretiennent à des processus décisionnels présente un degré de complexité particulier. D'une part, parce que l'architecture même du réseau semble favoriser la constitution de sphères publiques autonomes, davantage propices à une forme de renforcement de la société civile par l'exercice d'un contrepouvoir (Cardon, 2010). D'autre part, parce que les dispositifs participatifs mis en ligne dans le cadre de procédures institutionnalisées relèvent autant d'une mutation de la médiation politique que d'une évolution des pratiques de gouvernance (Monnoyer-Smith, 2011), et ne permettent que rarement aux participants qui y prennent part d'avoir un réel impact sur la décision finale (Vedel, 2003 ; Blondiaux et Cardon, 2006 ; Wojcik, 2011).

La plateforme Votre point de vue sur l'Europe, qui héberge les consultations permanentes de la Commission Européenne, constitue à ce titre un cas spécifique. Utilisée par les différents services de la Commission (les Directions Générales) dans le cadre de la préparation de directives et de règlements communautaires, son intégration au sein du processus législatif bénéficie d'une reconnaissance juridique, qui établit de fait un lien formel entre participation et décision (Badouard, 2010). Ces consultations constituent historiquement un levier d'influence pour les experts et les groupes d'intérêt (Michel, 2007), mais leur mise en ligne, à partir du début des années 2000, s'accompagne d'une implication accrue de citoyens « ordinaires », qui viennent 
côtoyer sur la plateforme des organisations non gouvernementales, des groupements professionnels et des institutions.

L'intérêt d'un tel terrain d'étude est alors de permettre une observation de la façon dont les spécificités du web, en termes d'interaction et de conduite d'actions collectives, contribuent ou non à faire évoluer les relations entre ces différentes catégories d'acteurs : l'espace de participation délimité par la plateforme permet-il une redistribution de certaines ressources d'influence sur les décisions de la Commission, « renforçant » ainsi les publics « faibles » constitués de l'ensemble des citoyens?

Pour répondre à cette question, nous mobiliserons les catégories d'analyse développées par Nancy Fraser (1992) et Erik O. Eriksen (2005; 2007) pour étudier les rapports des publics à la décision, en questionnant la pertinence d'un tel emprunt dans le cadre de l'étude de pratiques politiques en ligne. Nous préciserons d'abord les modalités d'articulation de cette plateforme au processus législatif européen, avant d'étudier les acteurs en présence et d'analyser, à travers le cas précis de la consultation sur l'initiative citoyenne européenne, leur rapport à la décision. Le but de cet article est d'appréhender les ressources d'ordre communicationnel dont disposent les différents publics, qui viennent s'ajouter à celles qui leur sont conférées par leur statut politique et leur parcours sociologique. Nous verrons ainsi que la « force » des publics réside également dans la maîtrise d'une « grammaire de la participation » à l'échelle européenne, et des modalités de collaboration entre les différentes catégories d'acteurs, elles-mêmes cadrées par les caractéristiques techniques de la plateforme.

\section{Les consultations de la Commission et leurs publics}

La mise en ligne de la plateforme Votre point de vue sur l'Europe s'inscrit directement dans le sillage de la « réforme de la gouvernance européenne », initiée au début des années 2000, et dont le but affiché est de favoriser un rapprochement de la société civile et des institutions communautaires. À cette époque la Commission dispose déjà d'un large éventail de mécanismes consultatifs (livres vert et blanc, comités ad hoc, panels d'entreprise ou encore auditions d'experts), qui favorisent la participation de publics plus ou moins organisés. Le recours à un site participatif relève alors d'une volonté d'élargir le spectre des acteurs concernés, en y intégrant notamment des citoyens « ordinaires » et des organisations non institutionnalisées.

La plateforme va ainsi centraliser l'ensemble des consultations publiques conduites par les différentes Directions Générales. Si ces consultations bénéficient d'une reconnaissance juridique, elles ne présentent pas de caractère contraignant : la Commission se réserve le droit de retenir ou de rejeter une proposition qui lui est faite, selon un impératif de pertinence dont elle juge seule de la teneur. L'impact d'une contribution sur un règlement ou une directive qui sera soumise au vote du Parlement dépend en fait de la stratégie mise en œuvre à travers la consultation. Celle-ci peut être de deux types : soit la consultation a pour but de mobiliser des sources d'expertise externes, c'est-à-dire des groupes d'intérêt et des organisations de la société civile extérieurs à un 
cercle bruxellois relativement restreint (Balme, Chabanet et Wright, 2002 ; Saurugger, 2006) - et cela à moindres frais, soit celle-ci vise à légitimer la décision en " mettant en scène » une participation de la société civile à sa construction. Ces deux stratégies ne sont certes pas incompatibles, à tel point que ces consultations ont été considérées comme une tentative de réconciliation des registres de légitimation « fonctionnelle » (ayant trait à l'efficacité du processus décisionnel) et « démocratique » (relatif à son caractère inclusif) de la Commission (Scharpf, 2000 ; BouzaGarcia, 2009). Pour autant, chaque Direction Générale qui organise une consultation valorise un objectif aux dépens d'un autre, cet agencement ayant un impact certain sur la prise en considération des contributions des participants. En effet, dans le premier cas, la Commission cherche à combler un défaut de connaissance sur un sujet spécifique, et se montre disposée à l'intégration d'une contribution dans un texte officiel, si celle-ci est jugée «pertinente ». Dans le second cas, en revanche, les contributions ont moins d'importance que le processus qui les produit : il s'agit principalement de communiquer sur la participation civique à la construction d'un texte, afin de l'auréoler d'une certaine légitimité démocratique (argument ayant son importance dans les jeux institutionnels internes entre Parlement et Commission), sans forcément chercher à intégrer les contenus des contributions.

Selon la segmentation établie par la Commission, trois grandes catégories de participants sont amenées à soumettre des propositions : les organisations de la société civile (catégorie qui englobe à la fois des groupes d'intérêt, des organisations non gouvernementales et des cabinets de conseil), les autorités publiques (c'est-à-dire des administrations, opérant à une échelle nationale, régionale ou locale), et les citoyens individuels (qui participent en leur nom propre). Cette catégorisation peut ici être rapprochée des notions de publics forts, segmentés et généraux, définies par Erik O. Eriksen (2005 ; 2007), dans la continuité des travaux de Nancy Fraser (1992). Dans un article où elle réalise une analyse critique du modèle habermassien de l'espace public, la politiste américaine développe en effet une typologie des publics en démocratie, en fonction du rapport que ceux-ci entretiennent aux processus décisionnels. Elle distingue ainsi des publics forts, institutionnalisés, dont les délibérations ont un impact direct sur les décisions politiques (comme les Parlements), et des publics faibles, éclatés au sein de la société civile, dont le rôle politique réside dans la formation d'une opinion publique qui se construit sinon contre, du moins en dehors du système politique représentatif. Chez Eriksen, cette typologie est adaptée au contexte européen à travers trois catégories dérivées. Les publics forts sont compris comme l'ensemble du personnel politique qui gravite autour des centres décisionnels et qui dispose de ressources spécifiques pour influencer la fabrique des politiques publiques. Les publics segmentés sont des publics organisés, spécialisés sur des thématiques particulières, qui interviennent de façon ponctuelle sur la prise de décision dans des situations de résolution de problèmes spécifiques (problem-solving), à propos desquels ils disposent d'une certaine expertise. Enfin, les publics généraux correspondent aux publics « faibles » de Fraser, constitués de citoyens « ordinaires » qui construisent une opinion publique, à la différence près qu'il leur est reconnu une certaine « force», 
dans la mesure où leur mobilisation ponctuelle dans l'espace public peut influencer une prise de décision (notamment via des manifestations, des grèves, ou des révoltes).

Le rapprochement de cette catégorisation avec celle de la Commission semble ici pertinent : les autorités publiques correspondent aux publics forts, proches des centres de décision, les organisations de la société civile relèvent des publics segmentés, disposant d'une expertise établie sur certaines thématiques, et les citoyens individuels constituent des publics généraux, exprimant un agrégat d'opinions personnelles. Si la correspondance existe entre les différentes typologies, précisons toutefois que les catégories sont poreuses, et que les acteurs, dans leur diversité, peuvent déborder leur cadre : par exemple, un citoyen disposant d'un bon niveau de connaissance sur un sujet spécifique appartiendra autant à un public segmenté que général, dans la mesure où son expertise personnelle peut être mobilisée dans un contexte de problem-solving ${ }^{1}$.

\section{Publics et décisions}

Si la plateforme Votre point de vue sur l'Europe constitue un espace dans lequel différents publics sont engagés dans une activité commune, cela ne signifie pas pour autant qu'ils disposent des mêmes ressources et leviers pour influencer la décision.

D'abord, les différentes catégories ne bénéficient pas de la même représentation, et les organisations de la société civile demeurent les interlocutrices privilégiées de la Commission : d'après un rapport du Conseil Économique, Social et Environnemental (CESE) français publié en 2009, celles-ci représentent $67,3 \%$ des participants, suivies par les institutions publiques $(18,5 \%)$ et les citoyens $(14,2 \%)$. Pour autant, cette répartition varie grandement d'une consultation à l'autre, selon deux facteurs principaux (Badouard, 2012a) : le premier a trait au sujet de la consultation lui-même, c'est-à-dire que plus un thème est technique et nécessite un certain niveau d'expertise, et plus la probabilité que des citoyens participent est faible ; le second est relatif à la traduction des documents accompagnant la consultation : plus cet effort de traduction est important, et plus grande est la probabilité que des citoyens individuels participent ${ }^{2}$.

Intéressons-nous maintenant aux modalités de prise en considération des différentes contributions par la Commission. Pour ce faire, nous prenons un cas particulier : la consultation menée en janvier 2010 par le Secrétariat Général sur l'initiative citoyenne européenne. Ce nouveau droit civique européen, introduit par le Traité de Lisbonne, permet à un million de citoyens signataires d'une même pétition, et originaires de différents États membres, d' « inviter » la Commission à conduire une initiative législative sur la question mise en lumière par la pétition. Avant son entrée en vigueur en avril 2012, la Commission voulait, par le biais de cette consultation, fixer les modalités de sa mise en œuvre. La consultation abordait donc un sujet assez général, ne nécessitant pas un haut niveau d'expertise technique. De plus, les documents liés étaient disponibles en 22 langues différentes, alors que la grande majorité des textes accompagnant les consultations ne sont disponibles qu'en anglais ${ }^{3}$. Dans ce cadre, la participation des citoyens a été importante, puisque $46,4 \%$ des 311 contributions reçues 
par la Commission provenaient d'individus, suivis par des organisations de la société civile $(41,3 \%)$ et des autorités publiques $(12,3 \%)$. Sous certaines conditions, certes restrictives, il semble donc que la mise en ligne des consultations de la Commission s'accompagne d'une ouverture du processus à de nouveaux acteurs, en permettant une participation de citoyens individuels.

Nous avons effectué un codage des propositions contenues dans ces 311 contributions, c'est-à-dire que nous les avons caractérisées et recensées afin d'évaluer quelles étaient les positions dominantes, en fonction des différentes catégories de publics. Nous avons ensuite croisé ces données avec différents documents officiels, afin de comparer ces positions à celles de la Commission et d'appréhender leur influence sur celles-ci. Ces documents sont les suivants : le «Livre Vert sur une initiative citoyenne européenne », qui cadre la consultation en présentant les dix questions auxquelles les participants doivent répondre, tout en explicitant pour chacune d'elle les positions de la Commission ; les résultats de la consultation, document dans lequel la Commission se livre à une synthèse des contributions ; le règlement final transmis au Conseil et au Parlement pour amendement, qui permet d'identifier les propositions retenues.

$\mathrm{Si}$, pour la plupart des questions présentées dans le Livre Vert, les positions de la Commission ont reçu un relatif soutien de la part des participants, certains points ont fait polémique. Face à l'expression d'avis divergents, la Commission a développé trois méthodes de gestion du dissensus : lorsque les propositions sont compatibles, elle les assemble en les ajustant ; lorsque celles-ci sont antagonistes, elle explore une voie alternative permettant de les satisfaire en partie ; lorsque celles-ci attaquent frontalement les options présentées dans le Livre Vert, ou font état de faits qui n'avaient pas été initialement pris en compte, la Commission réévalue ses positions ou y intègre des éléments soumis par les critiques. Si ces schémas ne s'observent pas pour chaque thématique développée dans la consultation, il n'en demeure pas moins que les propositions de la Commission ont connu une évolution importante entre la publication du Livre Vert et la transmission du règlement au Parlement et au Conseil, en partie sous l'effet des contributions soumises lors de la consultation.

Cette prise en considération est d'abord due à la stratégie des organisateurs. Comme nous le précisions plus haut, celle-ci se déploie selon une optique fonctionnelle : les fonctionnaires du Secrétariat général n'ayant qu'une faible connaissance des initiatives citoyennes, ils ont cherché par le biais de la consultation à profiter de l'expérience des organisations qui en conduisent et des pouvoirs publics qui les articulent à des processus décisionnels, à différentes échelles. Dans ce cadre, toutes les voix ne sont pas écoutées de la même façon et, de l'avis même des organisateurs ${ }^{4}$, les contributions des organisations et des autorités publiques bénéficient d'une plus grande attention, dans la mesure où elles sont considérées comme plus pertinentes au vu des besoins et attentes de la Commission.

\section{Grammaire de la participation et hybridation des publics}

Une contribution faisant état d'une certaine expérience en matière d'initiative citoyenne, ou 
d'une bonne connaissance du sujet, à même de souligner les défauts du règlement de la Commission et de proposer des solutions pour les pallier, était donc considérée comme davantage « utile », qu'une contribution exprimant un avis. Pour autant, cette « utilité », cette « pertinence », ne sont pas forcément liées au statut des acteurs. Par exemple, dans le cadre du processus d'étude des propositions que nous avons décrit plus haut, un citoyen disposant d'une expertise en la matière, ou une organisation exprimant un avis, serait davantage évalué à l'aune du contenu de sa contribution que de son statut lui-même.

La notion de « grammaire » peut ici nous être utile pour rendre compte de ce phénomène ${ }^{5}$. Nicolas Benvegnu et Mathieu Brugidou (2008) par exemple, parlent, dans un cas similaire au nôtre, de " grammaire de l'action publique », pour désigner une certaine habileté de participants à des procédures délibératives à formuler des opinions et des recommandations qui apparaissent comme crédibles aux yeux des décideurs. Autrement dit, maîtriser cette grammaire, c'est être capable de prendre en considération les contraintes de l'action publique, afin de proposer des recommandations jugées viables, réalistes et pertinentes, c'est-à-dire pouvant être mises en œuvre dans le cadre d'une politique publique. Certains des acteurs ayant contribué à la consultation sur l'initiative citoyenne sont des participants réguliers aux consultations de la Commission : ils sont inscrits au registre des représentants d'intérêt, qui constitue en quelques sortes un répertoire d'interlocuteurs pour la Commission, et disposent d'une bonne connaissance des mécanismes de l'action publique européenne. Face à d'autres participants, qui ne contribuent que ponctuellement en soumettant des avis, ils constituent des publics forts, dans le sens où ils sont plus à même de fournir des contributions en adéquation avec les attentes de la Commission, qui feront l'objet d'une attention plus soutenue de la part de celle-ci.

Ensuite, les différents publics s'activent au sein d'arènes plus ou moins bien connectées aux centres décisionnels. Les publics forts des Parlements ou des assemblées régionales par exemple, sont informés des consultations par le biais de services internes dédiés aux affaires européennes, alors que les milieux associatifs le sont davantage par des activités de veille et les citoyens « ordinaires » par le biais d'un relai de l'information dans les médias de masse ${ }^{6}$. Autrement dit, les arènes dans lesquelles évoluent les publics sont plus ou moins proches des espaces institutionnels de la Commission. La « distance» à parcourir pour participer, en ligne comme hors ligne, est plus importante pour les publics généraux que pour les publics segmentés, et pour les publics segmentés que pour les publics forts. Parce qu'ils entretiennent un rapport de proximité entre un public et un espace de décision, les canaux de communication dessinés entre la plateforme européenne et les arènes des publics constituent un autre élément leur conférant une certaine force.

Un dernier élément entrant en compte relève des possibilités techniques de collaboration sur la plateforme en ligne. En effet, si les différents publics évoluent dans des arènes hermétiques les unes aux autres, la plateforme participative constitue le point nodal où s'articulent ces arènes. Les publics y sont réunis dans un même 
espace, afin d'y réaliser une activité commune. Or, sur Votre point de vue sur l'Europe, il n'existe aucun moyen d'interaction entre les différents acteurs. La participation à une consultation de la Commission revient à envoyer par courriel sa contribution. Si l'échange avec la Commission est ainsi rendu possible, celui-ci reste vertical et les participants ne peuvent pas échanger entre eux. De plus les participants sont catégorisés selon leur statut : qu'ils soient citoyens, organisations, ou autorités publiques, ils adressent leur contribution via des courriels différents, et celles-ci sont publiées à différents endroits du site. Les voies de communication sont donc différenciées et hermétiques et les contributions sont, sinon hiérarchisées, du moins catégorisées en fonction du statut des participants.

On le voit, le design de la plateforme, et notamment la configuration des interactions et les pratiques de navigation influencent largement les modalités de constitution des publics en ligne. Les participants peuvent, une fois présents sur un site participatif, se retrouver dans trois situations différentes : une situation de co-présence, comme c'est le cas pour la plateforme étudiée ici, où les différents individus et organisations participent sans échanger ; une situation d'interaction, quand les participants peuvent échanger des informations les uns avec les autres; une situation d'hybridation, quand les participants sont invités à construire ensemble une contribution commune. Dans la dernière situation, les publics « faibles » se trouvent renforcés dans la mesure où la contribution commune, fournie à l'autorité publique, pousse celle-ci à procéder à un examen indifférencié au regard du statut des acteurs composant le public.
Les ressources communicationnelles contribuant en ligne à la force des publics relèvent dans le cas des consultations de la Commission Européenne de trois éléments : la maîtrise d'une grammaire de la participation européenne, qui permet aux participants de proposer des contributions jugées pertinentes par la Commission, car prenant en considération les contraintes de conduite des politiques publiques, et qui bénéficieront de ce fait d'une plus grande attention ; la connexion des arènes dans lesquelles évoluent les différents publics avec les espaces décisionnels, qui joue sur la fluidité de la circulation de l'information entre les publics et la Commission ; les caractéristiques techniques de la plateforme participative, notamment à travers les applications proposées, qui configurent les actions et interactions possibles, et permettent ou non d'hybrider les différents publics via des pratiques collaboratives.

\section{Conclusion}

L'analyse de la « force » de différents publics dans le cadre de procédures participatives institutionnalisées ne doit pas en rester à une mesure des impacts des différentes contributions sur la décision finale. En ligne comme hors ligne, les leviers de pouvoir dont disposent les participants relèvent avant tout de ressources attachées à un statut politique ou à un parcours sociologique. Pour autant, les ressources d'ordre communicationnel jouent également un rôle. Elles peuvent être directement liées aux ressources d'ordre politique ou sociologique, comme dans le cas de la maîtrise d'une grammaire de la participation à l'échelle européenne. Mais sur le web, deux éléments spécifiques doivent également faire l'objet d'une attention soutenue. D'une part, la 
connexion des espaces où évoluent les différents publics avec le centre décisionnel : celle-ci peut être plus ou moins directe, formelle et ouverte, influençant dès lors la représentation (quantitative) des différentes catégories de publics sur le site institutionnel. L'analyse de ces canaux de communication et de leurs caractéristiques est aujourd'hui facilitée par les techniques de « cartographie » du web (Badouard, 2012b), qui permettent de les représenter graphiquement. D'autre part, les caractéristiques techniques du site participatif, qui structurent les pratiques de collaboration entre les différents acteurs : lorsque les interactions entre les participants ne sont pas possibles, le site ne produit qu'une transposition en ligne des leviers de pouvoir des différents publics. À l'inverse, la production commune de propositions (via des applications techniques adaptées) peut favoriser la fusion des publics généraux, segmentés et forts au sein d'une même entité, dessinant de fait une voie pour le renforcement des publics « faibles ».

Condition nécessaire, l'application technique n'est cependant pas une condition suffisante : l'interaction et la construction commune doivent également correspondre aux intentions des acteurs visés. Si ceux-ci n'y voient pas d'intérêt stratégique, ils déserteront les espaces d'échange pour se livrer à des activités participatives individuelles. Dès lors, la voie dessinée pour ce renforcement, bordée par des possibles techniques d'un côté, et des stratégies d'acteurs de l'autre, semble bien étroite.

Cet article a bénéficié des retours de Françoise Massit-Folléa, qu'elle en soit ici remerciée.

\section{$R \cdot \dot{E} \cdot F \cdot \dot{E} \cdot R \cdot E \cdot N \cdot C \cdot E \cdot S$}

Romain BADOUARD, "Combining inclusion with impact on the decision? The Commission's online consultation on the European Citizens' Intiative", in Raphaël Kies et Patricia Nanz, European Citizens'Deliberation : a promising path for EU governance?, Dartmoth : Ashgate, à paraître en 2012 .

Romain BADOUARD, « La « cartographie du web », un outil méthodologique pour les SIC ? », Les Cahiers de la SFSIC, $\mathrm{n}^{\circ}$, printemps 2012, pp. 37-42.

Romain BADOUARD, "Pathways and Obstacles to e-Participation at the European level. A comparative analysis of the European Citizens' Consultation 2009 and the online consultations of the European Commission", Journal of eDemocracy and Open Government, 2(2), 2010, pp. 99-110. Richard BALME, Didier CHABANET, Vincent WRIGHT (dir.), L'action collective en Europe, Paris : Presses de Sciences Po, 2002.

Nicolas BENVEGNU, Mathieu BRUGIDOU, « Prendre la parole sur internet. Des dispositifs sociotechniques aux grammaires de la discussion », Réseaux, vol.4, n¹50, 2008, pp. 51-82. Luis BOUZA GARCIA, "Can segmented publics foster a general public sphere in the EU ? An example from the consultation practices of the European Commission", Observatorio (OBS) Journal, n9, 2009.

Loïc BLONDIAUX et Dominique CARDON, « Dispositifs participatifs », introduction au numéro éponyme de la revue Politix, vol.3, $\mathrm{n}^{\circ} 75$, 2006, pp. 3-9.

Michel CALLON, Pierre LASCOUMES, Yannick BARTHE, Agir dans un monde incertain. 
Essai sur la démocratie technique, Paris : Le Seuil, 2001.

Dominique CARDON, La démocratie internet : Promesses et Limites, coll. La République des idées, Paris : Éditions du Seuil, 2010.

Erik O. ERIKSEN, “Conceptualising European public spheres. General, segmented and strong publics", in Fossum, J., Schlesinger, P. (eds), The European Union and the Public Sphere: A communicative space in the making?, New York/ London : Routledge, 2007.

Erik O. ERIKSEN, “An emerging european public sphere", European Journal of Social Theory, Vol. 8, n³, London : Sage Publications, 2005, pp. 341-363.

Nancy FRASER, "Rethinking the Public Sphere : a contribution to a critique of actually existing democracy", Calhoun Craig (ed.), Habermas and the Public Sphere, Cambridge : MIT Press, 1992, pp. 109-142.

Cyril LEMIEUX, Le devoir et la grâce, Paris : Economica, 2009.

Hélène MICHEL, « La « société civile » dans la « gouvernance européenne ». Eléments pour une sociologie d'une catégorie politique », Actes de la recherche en sciences sociales, vol.1, n¹66-167, 2008, pp. 30-37.

Laurence MONNOYER-SMITH, Communication et délibération. Enjeux technologiques et mutations citoyennes, Paris : Hermès Lavoisier, 2010.

Sabine SAURUGGER, « Les groupes d'experts, une porte d'entrée de la société civile dans le processus décisionnel ? ", in Céline Bélot et Bruno Cautres (dir.), La vie démocratique de l'Union Européenne, Paris : La Documentation Française, 2006.

Fritz W. SCHARPF, Gouverner l'Europe, Paris :
Presses de Sciences Po, 2000.

Julien TALPIN, Schools of democracy. How ordinary citizens (sometimes) become competent in participatory budgeting institutions, Colchester : ECPR Press, 2011.

Thierry VEDEL, « L’idée de démocratie électronique. Origines, visions, questions », in Pascal Perrineau (dir.), Le désenchantement démocratique, La Tour d'Aigues : Éditions de l'Aube, 2003, pp. 243-266.

Stéphanie WOJCIK, «Prendre au sérieux la démocratie électronique. De quelques enjeux et controverses sur la participation politique en ligne », in Elsa Forey et Christophe Geslot (dir.) La démocratie électronique, Paris : L'Harmattan, coll. Questions contemporaines, 2011.

\section{Documents officiels}

Commission Européenne, Gouvernance Européenne, Un Livre Blanc, COM(2001) 428 final, Bruxelles, 2001.

Commission Européenne, Livre Vert sur une initiative citoyenne européenne, $\operatorname{COM}(2009)$ 622 final.

Commission Européenne, Outcome of the public consultation on the Green Paper on a European Citizens' Initiative. Accompanying document to the proposal for a Regulation of the European parliament and of the council on the citizens' initiative. SEC(2010) 370.

Commission Européenne, Proposal for a regulation of the European Parliament and of the Council on the citizens' initiative COM (2010) 119 final.

Conseil Économique, social et environnemental, Pour une participation active de la société civile aux consultations publiques européennes, 2009, Journal Officiel n²009-22. 


\section{$\mathrm{N} \cdot \mathrm{O} \cdot \mathrm{T} \cdot \mathrm{E} \cdot \mathrm{S}$}

1. On pense notamment ici aux collectifs de citoyens développant une expertise scientifique indépendante dans le cadre de controverses techniques, comme ils ont pu être décrits par M. Callon, P. Lascousmes et Y. Barthe (2001).

2. Si les textes officiels précisent qu'une contribution peut être envoyée dans l'une des 23 langues officielles reconnues par l'Union Européenne, les documents présentant les enjeux de la consultation et les modalités de réponse ne sont le plus souvent disponibles qu'en anglais.

3. Par exemple, sur les 93 consultations menées sur la plateforme en 2010, 75 n'étaient disponibles qu'en anglais.

4. Un membre de l'Unité Question Institutionnelle du Secrétariat Général, qui a participé à l'organisation de la consultation, évoquait, lors d'un entretien dans les locaux de la Commission en octobre 2010, la méthode d'analyse des contributions en ces termes : «On n'a pas voulu trop se baser sur la quantité (...) mais se concentrer sur les contributions où il y avait une vraie argumentation, et sur les organisations qui sont impliquées et qui ont déjà organisé des pétitions de masse ou des choses comme ça, des choses similaires à l'initiative, parce qu'ils ont de l'expérience, ils connaissent plus de choses sur le sujet, donc on regarde plus attentivement ces organisations, et les autorités publiques aussi, surtout en ce qui concerne la mise en ceuvre par les États Membres».

5. La notion de grammaire connait différentes utilisations en sociologie et en science politique : voir notamment (Lemieux, 2009) et (Talpin, 2011).

6. Cette affirmation se base sur un questionnaire envoyé à 154 des 311 participants à la consultation. 